\title{
Néron Models of Green-Griffiths-Kerr and log Néron Models
}

\author{
by
}

\author{
Tatsuki HaYAma
}

\begin{abstract}
For a variation of Hodge structure over a punctured disk, Green, Griffiths and Kerr introduced a Néron model which is a Hausdorff space that includes values of admissible normal functions. On the other hand, Kato, Nakayama and Usui introduced a Néron model as a logarithmic manifold using log mixed Hodge theory. This work constructs a homeomorphism between these two models.
\end{abstract}

2010 Mathematics Subject Classification: 14D07.

Keywords: Néron model, log mixed Hodge structure, admissible normal function, intermediate Jacobian.

\section{$\S 1$. Introduction}

Let $J \rightarrow \Delta^{*}$ be a family of intermediate Jacobians arising from a variation of polarized Hodge structure (VHS) of weight -1 with a unipotent monodromy on a punctured disk. By Carlson $[\mathrm{C}]$, the intermediate Jacobians are isomorphic to the extension groups of the Hodge structures, in the category of mixed Hodge structures (MHS). Then a section of $J \rightarrow \Delta^{*}$ is known as a variation of MHS (VMHS). A VMHS satisfying the admissibility condition [SZ] is called an admissible VMHS (AVMHS), and a section which gives an AVMHS is known as an admissible normal function (ANF) [Sa1].

For the VHS, Green, Griffiths and Kerr [GGK1] introduced the family $J^{\text {GGK }}$ $\rightarrow \Delta$ satisfying the following conditions:

- The family restricted to $\Delta^{*}$ is $J \rightarrow \Delta^{*}$;

- The fiber over 0 is a complex Lie group;

Communicated by M. Kashiwara. Received March 23, 2010. Revised August 4 and September 8, 2010 .

T. Hayama: Department of Mathematics, National Taiwan University, Taipei 106, Taiwan; e-mail: t-hayama@math.ntu.edu.tw

(C) 2011 Research Institute for Mathematical Sciences, Kyoto University. All rights reserved. 
- Any ANF is a section of $J^{\mathrm{GGK}} \rightarrow \Delta$;

- $J^{\mathrm{GGK}}$ is a Hausdorff space.

The total space $J^{\text {GGK }}$ is called a Néron model. Here, $J^{\text {GGK }}$ is simply a topological space. The authors of [GGK1] propose "doing geometry" on Néron models.

In contrast, Kato, Nakayama and Usui constructed Néron models via a log mixed Hodge theory. To explain their work, we describe $J \rightarrow \Delta^{*}$ by another formulation. Let $\Delta^{*} \rightarrow \Gamma \backslash D$ be the period map arising from the VHS. The family of intermediate Jacobians can then be obtained as the fiber product

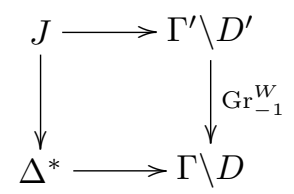

where $D^{\prime}$ and $\Gamma^{\prime}$ are used for the MHS corresponding to the intermediate Jacobians.

Kato, Nakayama and Usui [KNU1] extended the above diagram. First, Kato and Usui $[\mathrm{KU}]$ stated that the period map can be extended to

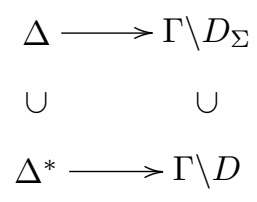

where $\Sigma$ is the fan of nilpotent cones arising from the monodromy of the VHS. Here a boundary point of $\Gamma \backslash D_{\Sigma}$ is a nilpotent orbit, which approximates the period map given by Schmid [Sc]. The main theorem of [KU] states that $\Gamma \backslash D_{\Sigma}$ is a logarithmic manifold and that it is a moduli space of log (pure) Hodge structures.

Next, an ANF is written as

$$
\Delta^{*} \rightarrow \Gamma^{\prime} \backslash D^{\prime} .
$$

Kato, Nakayama and Usui [KNU2] give the fan $\Sigma^{\prime}$, by means of which this map can be extended to

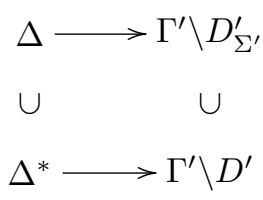

Similarly to the pure case $[\mathrm{KU}]$, a boundary point of $\Gamma^{\prime} \backslash D_{\Sigma^{\prime}}^{\prime}$ is a nilpotent orbit, which approximates the ANF by the method proposed by Pearlstein $[\mathrm{P}]$. The main theorem of [KNU2] states that $\Gamma^{\prime} \backslash D_{\Sigma^{\prime}}^{\prime}$ is a logarithmic manifold and a moduli space of $\log$ mixed Hodge structures. 
Finally, they define the $\log$ Néron model $J^{\mathrm{KNU}}$ as the fiber product

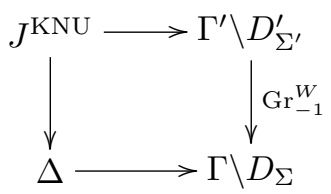

in the category of logarithmic manifolds. We remark that $J^{\mathrm{KNU}}$ is not only a topological space but also has a geometric structure as a logarithmic manifold.

However, [KNU1] does not show the relationship between $J^{\mathrm{GGK}}$ and $J^{\mathrm{KNU}}$. In fact, $\S 8.2$ of [KNU1] states that the relationship is apparently unknown between $J^{\mathrm{KNU}}$ and the Néron model constructed by Green, Griffiths and Kerr. Our main aim is to solve this problem.

Theorem 1.1 (Theorem 5.1). $J^{\mathrm{GGK}}$ is homeomorphic to $J^{\mathrm{KNU}}$.

We explain the idea of the proof. By using the liftings in (4.1) and (4.6), we construct a bijective map between the two sets (in Proposition 4.4). In Section 5, we show that this map is a homeomorphism. The diagram (3.5) and the admissibility condition $((2.6)$ or $(2.10))$ play important roles in the proof.

\section{§2. Preliminaries}

In this section, we recall the definitions of the Néron models given in [GGK1] and [KNU2]. Let $\left(\mathcal{H}_{\mathbb{Z}}, \mathcal{F}, \nabla\right)$ be a variation of polarized Hodge structure of weight -1 over a punctured disk $\Delta^{*}$, where $\mathcal{H}_{\mathbb{Z}}$ is a local system, $\mathcal{F}$ is a filtration of a locally free sheaf $\mathcal{H}:=\mathcal{H}_{\mathbb{Z}} \otimes \mathcal{O}_{\Delta^{*}}$ and $\nabla$ is a Gauss-Manin connection. We assume that the monodromy transformation $T$ is unipotent.

\section{§2.1. Families of intermediate Jacobians}

Let $(H, F)$ be the total space of the vector bundle corresponding to the VHS $(\mathcal{H}, \mathcal{F})$. The intermediate Jacobian over $s \in \Delta^{*}$ is defined as

$$
J_{s}:=F_{s}^{0} \backslash H_{s} / \mathcal{H}_{\mathbb{Z} ; s}
$$

where the subscript $s$ denotes the fiber (or stalk) over $s$. By Carlson [C], we have the isomorphism

$$
\operatorname{Ext}_{\mathrm{MHS}}^{1}\left(\mathbb{Z}(0), H_{s}\right) \cong J_{s}
$$

where $\mathbb{Z}(0)$ is Tate's Hodge structure.

We describe the family of intermediate Jacobians $J \rightarrow \Delta^{*}$ using the MHS in (2.1). Fix a reference point $s_{0} \in \Delta^{*}$. For the PHS $H_{s_{0}}=\left(H_{\mathbb{Z}}, F_{s_{0}},\langle\rangle,\right)$ over $s_{0}$, 
we take a MHS $H^{\prime}$ which represents an extension class in $\operatorname{Ext}_{\mathrm{MHS}}^{1}\left(\mathbb{Z}(0), H_{s_{0}}\right)$. Let $D$ (resp. $D^{\prime}$ ) be the period domain for the type of $H_{s_{0}}$ (resp. $H^{\prime}$ ), defined in [G] (resp. [U]). The VHS gives the period map $\phi: \Delta^{*} \rightarrow \Gamma \backslash D$ where $\Gamma$ is the monodromy group. Here we may write

$$
\Gamma=\left\{T^{n} \in \operatorname{Aut}\left(H_{\mathbb{Z}}\right) \mid n \in \mathbb{Z}\right\} .
$$

Then the family of intermediate Jacobians is obtained by the following Cartesian diagram:

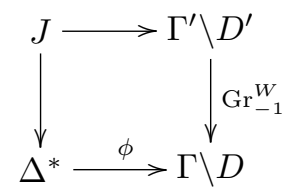

where $\Gamma^{\prime}:=\left\{T^{\prime} \in \operatorname{Aut}\left(H_{\mathbb{Z}}^{\prime}\right)\left|T^{\prime}\right|_{\operatorname{Aut}\left(H_{\mathbb{Z}}\right)} \in \Gamma\right\}$.

We now review some properties of the period domains $D$ and $D^{\prime}$. Let $\check{D}$ (resp. $\left.\check{D}^{\prime}\right)$ be the compact dual of $D$ (resp. $D^{\prime}$ ), defined in [G] (resp. [U]). From $[\mathrm{G}, \S 4]$ (resp. [U, §2]) we have the following properties for the pure case (resp. for some mixed case including the case of $D^{\prime}$ ):

Proposition 2.1. Let $G_{A}:=\operatorname{Aut}\left(H_{A},\langle\rangle,\right)\left(\right.$ resp. $\left.G_{A}^{\prime}:=\operatorname{Aut}\left(H_{A}^{\prime},\langle,\rangle_{\bullet}\right)\right)$ for $A=\mathbb{Z}, \mathbb{R}, \mathbb{Q}, \mathbb{C}$. Then

(1) $G_{\mathbb{R}}\left(\right.$ resp. $\left.G_{\mathbb{R}}^{\prime}\right)$ acts on $D\left(\right.$ resp. $\left.D^{\prime}\right)$ transitively;

(2) $G_{\mathbb{C}}\left(\right.$ resp. $\left.G_{\mathbb{C}}^{\prime}\right)$ acts on $\check{D}\left(\right.$ resp. $\left.\check{D}^{\prime}\right)$ transitively;

(3) any subgroup of $G_{\mathbb{Z}}\left(\right.$ resp. $\left.G_{\mathbb{Z}}^{\prime}\right)$ acts on $D\left(\right.$ resp. $\left.D^{\prime}\right)$ properly discontinuously.

Since $H^{\prime}$ is an extension of $H_{s_{0}}$ by $\mathbb{Z}(0)$, we have the exact sequence

$$
0 \rightarrow H_{\mathbb{Z}} \stackrel{i}{\rightarrow} H_{\mathbb{Z}}^{\prime} \stackrel{j}{\rightarrow} \mathbb{Z} \rightarrow 0
$$

of $\mathbb{Z}$-modules. We fix $e \in H_{\mathbb{Z}}^{\prime}$ such that $j(e)=1$. Then we may write

$$
H_{\mathbb{Z}}^{\prime} \cong H_{\mathbb{Z}} \oplus \mathbb{Z} e .
$$

We set

$$
\mathfrak{h}:=\left\{X \in \operatorname{End}\left(H_{\mathbb{C}}^{\prime}\right)|X|_{\operatorname{End}\left(H_{\mathbb{C}}\right)}=0, X(e) \in H_{\mathbb{C}}\right\}
$$

Proposition 2.2 ([U, Theorem 2.16]). $\mathrm{Gr}_{-1}^{W}: \check{D}^{\prime} \rightarrow \check{D}$ is a fiber bundle with fiber $\mathfrak{h} /(\mathfrak{h} \cap \mathfrak{b})$. Here, $\mathfrak{b}$ is the Lie algebra of an isotropy subgroup of $G_{\mathbb{C}}$. 


\section{$\S 2.2$. Normal functions and the identity components}

We first define the following sheaves over $\Delta^{*}$ :

$$
\mathcal{J}:=\mathcal{F}^{0} \backslash \mathcal{H} / \mathcal{H}_{\mathbb{Z}}, \quad \mathcal{J}_{\nabla}:=\left\{\begin{array}{l|l}
\nu \in \mathcal{J} & \begin{array}{l}
\nabla \tilde{\nu} \in \mathcal{F}^{-1} \otimes \Omega^{1} \\
\text { for any local lifting } \tilde{\nu}
\end{array}
\end{array}\right\}
$$

Since the monodromy is unipotent, we have the Deligne extension $\left(\mathcal{H}_{e}, \mathcal{F}_{e}\right)$. Then we define the following sheaves over $\Delta$ :

$$
\mathcal{J}_{e}:=\mathcal{F}_{e}^{0} \backslash \mathcal{H}_{e} / j_{*} \mathcal{H}_{\mathbb{Z}}, \quad \mathcal{J}_{e, \nabla}:=\mathcal{J}_{e} \cap j_{*} \mathcal{J}_{\nabla}
$$

where $j: \Delta^{*} \hookrightarrow \Delta$. A section of $\mathcal{J}_{e, \nabla}$ is called a normal function (NF).

Secondly we define a space that includes values of NF according to [GGK1, $\S$ II.A]. Let $\left(H_{e}, F_{e}\right)$ be the total space of the vector bundles corresponding to $\left(\mathcal{H}_{e}, \mathcal{F}_{e}\right)$. Since these vector bundles are trivial, we have a trivialization

$$
F_{e}^{n} \cong \Delta \times F_{e ; 0}^{n} .
$$

Since $\left(F_{e ; 0}, W(N)\right)$ is a MHS [Sc], we have the Deligne decomposition $H_{e ; 0}=$ $\bigoplus_{p, q} I^{p, q}$. It induces

$$
F_{e ; 0}^{0} \backslash H_{e ; 0} \cong \bigoplus_{p<0} I^{p, q}=: V
$$

By the trivialization (2.3), we may write

$$
F_{e}^{0} \backslash H_{e} \cong \Delta \times V
$$

We define the quotient space

$$
J^{Z}:=F_{e}^{0} \backslash H_{e} / \sim
$$

where the equivalence relation $\sim$ is given by equating two elements $(s, x),\left(s^{\prime}, x^{\prime}\right) \in$ $\Delta \times V \cong F_{e}^{0} \backslash H_{e}$ if and only if $s=s^{\prime}$ and $x-x^{\prime} \in j_{*} \mathcal{H}_{\mathbb{Z} ; s}$. We call it the Zucker space.

The Zucker space $J^{Z}$ includes values of NF. However, $J^{Z}$ is not generally a Hausdorff space (cf. [GGK1, II.B.8]). Hence, [GGK1] defines a subspace of $J^{Z}$ so that it is a Hausdorff space including values of NF.

Definition 2.3 ([GGK1, II.A.9]). Let

$$
W:=\{(s, x) \in \Delta \times V \mid x \in \operatorname{Ker}(N) \text { if } s=0\} .
$$

The identity component of the Néron model is the subset $J^{\mathrm{GGK}, 0}:=W / \sim$ of the Zucker space $J^{Z}$. Here the topology on $J^{\mathrm{GGK}, 0}$ is induced from the strong topology of $W$ in $\Delta \times V$ [KU, §3.1.1]. 
The identity component has the following property:

Proposition 2.4 ([GGK1, II.A.9]). For a $N F \nu, \nu(0) \in J_{0}^{\mathrm{GGK}, 0}$.

Remark 2.5. In [GGK1], the definition of the topology on $J^{\mathrm{GGK}, 0}$ seems to be unclear (a remark after [GGK1, Theorem II.A.9] states "This topology is modeled on the 'strong topology' in [KU]"). In this paper, we use the strong topology on $W \subset \Delta \times V$. Saito [Sa2] proves the Hausdorff property in the case of the ordinary topology.

\section{§2.3. Admissible normal functions and Néron models}

In accord with [GGK1, §II.B], we define the sheaf

(2.6) $\tilde{\mathcal{J}}_{e, \nabla}:=\left\{\begin{array}{l|l}\nu \in j_{*} \mathcal{J}_{\nabla} & \begin{array}{l}\tilde{\nu} \text { has a logarithmic growth as a section of } \check{\mathcal{F}}_{e}^{0}, \\ (T-I) \tilde{\nu} \in(T-I) \mathcal{H}_{\mathbb{Q}} \cap \mathcal{H}_{\mathbb{Z}} \text { for any local lifting } \tilde{\nu}\end{array}\end{array}\right\}$

where we denote the analytic continuation around the origin 0 of $\tilde{\nu}$ by $(T-I) \tilde{\nu}$. A section of $\tilde{\mathcal{J}}_{e, \nabla}$ is called an admissible normal function (ANF). By definition, we have the following exact sequence of sheaves:

$$
0 \rightarrow \mathcal{J}_{e, \nabla} \stackrel{i}{\rightarrow} \tilde{\mathcal{J}}_{e, \nabla} \stackrel{j}{\rightarrow} G_{0} \rightarrow 0
$$

Here $G_{0}$ is the skyscraper sheaf supported at 0 , whose stalk is

$$
G:=\frac{(T-I) H_{\mathbb{Q}} \cap H_{\mathbb{Z}}}{(T-I) H_{\mathbb{Z}}}
$$

We define the abelian group

$$
J_{s}^{\mathrm{GGK}}:=\frac{J_{s}^{\mathrm{GGK}, 0} \times \tilde{\mathcal{J}}_{e, \nabla ; s}}{\left\{\left(\nu(s),[i(\nu)]_{s}\right) \mid \nu \in \mathcal{J}_{e, \nabla}\right\}}
$$

where $[i(\nu)]_{s}$ is the germ at $s \in \Delta$. Since $\mathcal{J}_{e, \nabla ; s}$ is a divisible abelian group (i.e., for every positive integer $n$ and every $\nu \in \mathcal{J}_{e, \nabla ; s}$ there exists $\mu \in \mathcal{J}_{e, \nabla ; s}$ such that $n \mu=\nu)$ and $G$ is a finite group, the exact sequence of the stalks of (2.7) is split [GGK1, II.B.11]. Then we have the isomorphism

$$
J_{s}^{\mathrm{GGK}} \cong \begin{cases}J_{s}^{\mathrm{GGK}, 0} & \text { if } s \neq 0 \\ J_{s}^{\mathrm{GGK}, 0} \times G & \text { if } s=0 .\end{cases}
$$

Definition 2.6 ([GGK1, II.B.9]). The Néron model of Green-Griffiths-Kerr is the topological space

$$
J^{\mathrm{GGK}}:=\bigsqcup_{s \in \Delta} J_{s}^{\mathrm{GGK}} .
$$


Here the topology on $J^{\text {GGK }}$ is defined by the open sets

$$
S(\nu):=\left\{\left((s, x),[\nu]_{s}\right) \in J^{\mathrm{GGK}} \mid(s, x) \in S\right\}
$$

where $S$ is an open set of $J^{\mathrm{GGK}, 0}$ and $\nu$ is an ANF.

Example 2.7 (Classical case). Let $\bar{f}: \bar{E} \rightarrow \Delta$ be a degenerating family of elliptic curves of Kodaira type $I_{n}$. For the restriction $f: E \rightarrow \Delta^{*}$, we have the local system $\mathcal{H}_{\mathbb{Z}}:=R^{1} f_{*} \mathbb{Z}$ and the filtration $\mathcal{F}^{p}=R^{1} f_{*}\left(\Omega_{E / \Delta^{*}}^{\geq p}\right)$. Here $\left(\mathcal{H}_{\mathbb{Z}}, \mathcal{F}\right)$ is a VHS over $\Delta^{*}$ with a unipotent monodromy. In this case,

$$
J_{0}^{\mathrm{GGK}, 0} \cong \mathbb{G}_{\mathrm{m}}, \quad G \cong \mathbb{Z} / n \mathbb{Z}
$$

twisting $\left(\mathcal{H}_{\mathbb{Z}}, \mathcal{F}\right)$ into the VHS of weight -1 .

\section{§2.4. A nonclassical example}

We give an example where the Néron model is not an analytic space. Our two sources, [GGK2, §III.A] and [KNU1, §9], deal with special situations of this kind.

Let $Y$ be a singular $K 3$ surface (i.e., $\rho(Y)=20$ ) and $\bar{f}: \bar{E} \rightarrow \Delta$ be a degenerating family of elliptic curves of Kodaira type $I_{n}$. By the Shioda-Inose correspondence [SI], for a transcendental basis $\left\{t_{1}, t_{2}\right\}$ of $H^{2}(Y)$, the intersection form is represented as

$$
\left(t_{i} \cdot t_{j}\right)_{i, j}=\left(\begin{array}{cc}
2 a & b \\
b & 2 c
\end{array}\right)
$$

where $a, b, c \in \mathbb{Z}, a, c>0$ and $b^{2}-4 a c<0$. We assume that $a=m$, where $m$ is a square free positive integer, and that $b=0$ and $c=1$. We take a symplectic basis $\{\alpha, \beta\}$ of $H^{1}\left(E_{s}\right)$ for $s \neq 0$ such that the monodromy action is

$$
\alpha \mapsto \alpha+n \beta, \quad \beta \mapsto \beta
$$

If we set

$$
e_{1}=t_{1} \times \alpha, \quad e_{2}=t_{2} \times \alpha, \quad e_{3}=\frac{t_{1}}{2 m} \times \beta, \quad e_{4}=\frac{t_{2}}{2} \times \beta
$$

in $H^{3}\left(Y \times E_{s}, \mathbb{Q}\right)$, then the intersection form is represented as

$$
\left(e_{i} \cdot e_{j}\right)_{i, j}=\left(\begin{array}{cc}
0 & I \\
-I & 0
\end{array}\right) .
$$

For the family $g:=f \circ \mathrm{pr}_{2}: Y \times E \rightarrow \Delta^{*}$, we consider the local system $\mathcal{H}_{\mathbb{Z}} \subset R^{3} g_{*} \mathbb{Q}$ such that $\mathcal{H}_{\mathbb{Z}, s}=\sum_{i} \mathbb{Z} e_{i}$ and the filtration $\mathcal{F}^{p}$ induced from $R^{3} g_{*}\left(\Omega_{Y \times E / \Delta^{*}}^{\geq p}\right)$. Then $\left(\mathcal{H}_{\mathbb{Z}}, \mathcal{F}\right)$ is a VHS and a fiber $\left(\mathcal{H}_{\mathbb{Z}, s}, F_{s}\right)$ is a PHS of 
weight -1 where $h^{1,-2}=h^{0,-1}=h^{-1,0}=h^{-2,1}=1$, twisting it into the VHS of weight -1 . The monodromy transformation reads

$$
T=\left(\begin{array}{ccc}
I_{2} & 0 \\
2 m n & 0 & \\
0 & 2 n & I_{2}
\end{array}\right)
$$

By [KU, §12.3], the limiting MHS is described by the following Hodge diamond:

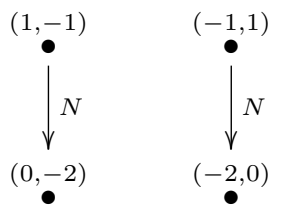

Then

$$
J_{0}^{\mathrm{GGK}, 0} \cong I^{-2,0} / j_{*} \mathcal{H}_{\mathbb{Z} ; 0}, \quad G \cong \mathbb{Z} / 2 m n \mathbb{Z} \times \mathbb{Z} / 2 n \mathbb{Z} .
$$

In this case, the dimension of $J_{0}^{\mathrm{GGK}, 0}$ is smaller than the dimension of a general fiber and $J^{\mathrm{Z}}$ is not a Hausdorff space (cf. [KNU1, §9]).

\section{§2.5. Moduli spaces of log Hodge structures and log Néron models}

Let $\mathfrak{g}_{A}$ (resp. $\mathfrak{g}_{A}^{\prime}$ ) be the Lie algebra of $G_{A}$ (resp. $\left.G_{A}^{\prime}\right)$ for $A=\mathbb{R}, \mathbb{C}$. Writing $\sigma=\mathbb{R}_{\geq 0} N$ in $\mathfrak{g}_{\mathbb{R}}$ with $N=\log (T)$, we define the fan $\Sigma:=\{\{0\}, \sigma\}$ and the set

$$
D_{\Sigma}=\left\{(\sigma, Z) \mid \sigma \in \Sigma, Z=\exp \left(\sigma_{\mathbb{C}}\right) F \text { is a } \sigma \text {-nilpotent orbit }\right\} .
$$

By $[\mathrm{KU}]$, the period map $\phi: \Delta^{*} \rightarrow \Gamma \backslash D$ extends to the $\log$ period map $\phi: \Delta \rightarrow$ $\Gamma \backslash D_{\Sigma}$.

Following [KNU1], we define the fan

$$
\Sigma^{\prime}:=\left\{\begin{array}{l|l}
\mathbb{R}_{\geq 0} N^{\prime} & \begin{array}{l}
N^{\prime} \in \text { End } H_{\mathbb{Q}}^{\prime},\left.N^{\prime}\right|_{\text {End } H_{\mathbb{Q}}}=N, \\
N^{\prime}(e)=N(a) \text { for some } a \in H_{\mathbb{Q}} \text { such that }(T-I) a \in H_{\mathbb{Z}}
\end{array}
\end{array}\right\} .
$$

Proposition 2.8. Let $\sigma^{\prime} \in \Sigma^{\prime}$. Then there exists a generator $N^{\prime} \in$ End $H_{\mathbb{Q}}^{\prime}$ of $\sigma^{\prime}$ such that $\exp \left(N^{\prime}\right) \in \Gamma^{\prime}$, and $\operatorname{Ad}(\gamma) \sigma^{\prime} \in \Sigma^{\prime}$ for $\gamma \in \Gamma^{\prime}$. Therefore $\Gamma^{\prime}$ is strongly compatible with $\Sigma^{\prime}$.

Proof. By definition, a generator of $\sigma^{\prime}$ is written as

$$
\left(\begin{array}{cc}
N & N a \\
0 & 0
\end{array}\right)
$$

with respect to the decomposition (2.2) for some $a \in H_{\mathbb{Q}}$. Moreover, we may write

$$
\Gamma^{\prime}=\left\{\left(\begin{array}{cc}
T^{n} & b \\
0 & 1
\end{array}\right) \mid b \in H_{\mathbb{Z}}, n \in \mathbb{Z}\right\} .
$$


Since $(T-I) a \in H_{\mathbb{Z}}$, we have

$$
\exp \left(N^{\prime}\right)=\left(\begin{array}{cc}
T & (T-I) a \\
0 & 1
\end{array}\right) \in \Gamma^{\prime}
$$

For $\gamma=\left(\begin{array}{cc}T^{n} & b \\ 0 & 1\end{array}\right) \in \Gamma^{\prime}$, we have

$$
\operatorname{Ad}(\gamma) N^{\prime}=\left(\begin{array}{cc}
N & N\left(T^{n} a-b\right) \\
0 & 0
\end{array}\right)
$$

Since $(T-I)\left(T^{n} a-b\right) \in H_{\mathbb{Z}}$, it follows that $\operatorname{Ad}(\gamma) N^{\prime} \in \Sigma^{\prime}$.

Similarly to $(2.9), D_{\Sigma^{\prime}}^{\prime}$ is defined as the set of nilpotent orbits [KNU2, $\left.\S 2.1 .3\right]$. Using the above proposition, we define the action

$$
\Gamma^{\prime} \times D_{\Sigma^{\prime}}^{\prime} \rightarrow D_{\Sigma^{\prime}}^{\prime} ; \quad\left(\gamma,\left(\sigma^{\prime}, Z\right)\right) \mapsto\left(\operatorname{Ad}(\gamma) \sigma^{\prime}, \gamma Z\right)
$$

and the orbit space $\Gamma^{\prime} \backslash D_{\Sigma^{\prime}}^{\prime}$.

The geometric structure on $\Gamma^{\prime} \backslash D_{\Sigma^{\prime}}^{\prime}$ is defined in [KNU2, §2.2.2]. For a nilpotent cone $\sigma^{\prime} \in \Sigma^{\prime}$, we define the monoid

$$
\Gamma^{\prime}\left(\sigma^{\prime}\right):=\Gamma^{\prime} \cap \exp \left(\sigma^{\prime}\right)
$$

and the toric variety

$$
\text { toric }_{\sigma^{\prime}}:=\operatorname{Spec}\left(\mathbb{C}\left[\Gamma^{\prime}\left(\sigma^{\prime}\right)^{\vee}\right]\right)_{\text {an }} \cong \mathbb{C} .
$$

Moreover, we define the analytic space

$$
\check{E}_{\sigma^{\prime}}^{\prime}:=\text { toric }_{\sigma^{\prime}} \times \check{D}^{\prime}
$$

and the subspace

$$
E_{\sigma^{\prime}}^{\prime}=\left\{\begin{array}{l|l}
(s, F) \in \check{E}_{\sigma^{\prime}}^{\prime} & \begin{array}{l}
\exp \left(l(s) N^{\prime}\right) F \in D^{\prime} \text { if } s \neq 0, \\
\exp \left(\sigma_{\mathbb{C}}^{\prime}\right) F \text { is a nilpotent orbit if } s=0
\end{array}
\end{array}\right\}
$$

where $l(s)$ is a branch of $(2 \pi i)^{-1} \log (s)$. The topology on $E_{\sigma^{\prime}}^{\prime}$ is the strong topology in $\check{E}_{\sigma^{\prime}}^{\prime}$. We then have the map

$$
E_{\sigma^{\prime}}^{\prime} \stackrel{p_{1}^{\prime}}{\rightarrow} \Gamma^{\prime}\left(\sigma^{\prime}\right)^{\mathrm{gp}} \backslash D_{\sigma^{\prime}}^{\prime} \stackrel{p_{2}^{\prime}}{\rightarrow} \Gamma^{\prime} \backslash D_{\Sigma^{\prime}}^{\prime} ; \quad(s, F) \mapsto \begin{cases}\left(0, \exp \left(l(s) N^{\prime}\right) F\right) & \text { if } s \neq 0, \\ \left(\sigma^{\prime}, \exp \left(\sigma_{\mathbb{C}}^{\prime}\right) F\right) & \text { if } s=0 .\end{cases}
$$

The geometric structure on $\Gamma^{\prime} \backslash D_{\Sigma^{\prime}}^{\prime}$ is induced from $E_{\sigma^{\prime}}^{\prime}$ locally through this map. Moreover Kato, Nakayama and Usui announced the following theorem:

Theorem 2.9 ([KNU2, Main Theorem]). Similarly to the pure case ([KU, Main Theorem]), the following holds: 
(1) $E_{\sigma^{\prime}}^{\prime}, \Gamma^{\prime}\left(\sigma^{\prime}\right)^{\mathrm{gp}} \backslash D_{\sigma^{\prime}}^{\prime}$ and $\Gamma^{\prime} \backslash D_{\Sigma^{\prime}}^{\prime}$ are logarithmic manifolds;

(2) $E_{\sigma^{\prime}}^{\prime} \rightarrow \Gamma^{\prime}\left(\sigma^{\prime}\right)^{\mathrm{gp}} \backslash D_{\sigma^{\prime}}^{\prime}$ is a $\sigma_{\mathbb{C}^{\prime}}^{\prime}$-torsor;

(3) $\Gamma^{\prime}\left(\sigma^{\prime}\right)^{\mathrm{gp}} \backslash D_{\sigma^{\prime}}^{\prime} \rightarrow \Gamma^{\prime} \backslash D_{\Sigma^{\prime}}^{\prime}$ is locally an isomorphism;

(4) $\Gamma^{\prime} \backslash D_{\Sigma^{\prime}}^{\prime}$ is a moduli space of log mixed Hodge structures.

Definition 2.10 ([KNU1, $\S 7]$ ). The log Néron model is the fiber product

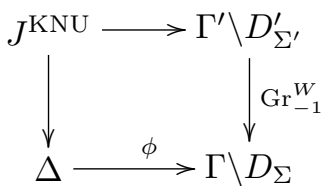

in the category $\mathcal{B}(\log )[\mathrm{KU}, 3.2 .4]$.

We describe the topology on $J^{\mathrm{KNU}}$. We now have the following diagram:

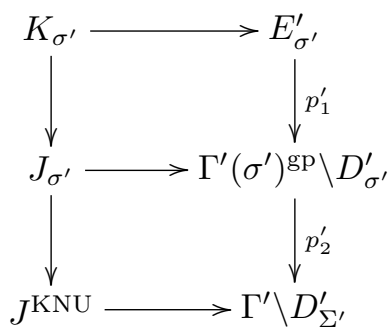

where $K_{\sigma^{\prime}}$ and $J_{\sigma^{\prime}}$ are the fiber products in $\mathcal{B}(\log )$. Here the topology on $K_{\sigma^{\prime}}$ is the strong topology in $\Delta \times \check{E}_{\sigma^{\prime}}^{\prime}$. The topological structures of $J_{\sigma^{\prime}}\left(\right.$ resp. $\left.J^{\mathrm{KNU}}\right)$ are induced from $K_{\sigma^{\prime}}$ through the morphism $K_{\sigma^{\prime}} \rightarrow J_{\sigma^{\prime}}\left(\operatorname{resp} . K_{\sigma^{\prime}} \rightarrow J^{\mathrm{KNU}}\right)$.

\section{§3. The relationship between $E_{\sigma} \rightarrow \Gamma(\sigma)^{\mathrm{gp}} \backslash D_{\sigma}$ and $E_{\sigma^{\prime}}^{\prime} \rightarrow \Gamma^{\prime}\left(\sigma^{\prime}\right)^{\mathrm{gp}} \backslash D_{\sigma^{\prime}}^{\prime}$}

The results of this section can be easily verified using [KNU2]; however the details will be useful in later sections. In the following section, we regard $E_{\sigma}$ (resp. $E_{\sigma^{\prime}}^{\prime}$ ) as a topological space whose topology is the strong topology in $\check{E}_{\sigma}$ (resp. $\check{E}_{\sigma^{\prime}}^{\prime}$ ).

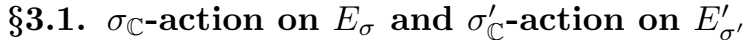

For $\sigma=\mathbb{R}_{\geq 0} N \in \Sigma$, we define the algebraic torus

$$
\operatorname{torus}_{\sigma}:=\operatorname{Spec}\left(\mathbb{C}\left[\Gamma(\sigma)^{\vee g p}\right]\right)_{\mathrm{an}} \cong \mathbb{G}_{\mathrm{m}}
$$

and the toric variety

$$
\operatorname{torus}_{\sigma}:=\operatorname{Spec}\left(\mathbb{C}\left[\Gamma(\sigma)^{\vee}\right]\right)_{\text {an }} \cong \mathbb{C}
$$


We then have the surjective map

$$
\sigma_{\mathbb{C}} \rightarrow \operatorname{torus}_{\sigma} ; \quad w N \mapsto \exp (2 \pi \sqrt{-1} w),
$$

which induces the action

$$
\sigma_{\mathbb{C}} \times \text { toric }_{\sigma} \rightarrow \text { toric }_{\sigma} ; \quad(w N, s) \mapsto \exp (2 \pi \sqrt{-1} w) s .
$$

For $\sigma^{\prime}=\mathbb{R}_{\geq 0} N^{\prime} \in \Sigma^{\prime}$, the $\sigma_{\mathbb{C}^{-}}^{\prime}$ action on toric ${ }_{\sigma^{\prime}}$ is defined similarly.

By the correspondence $N \leftrightarrow N^{\prime}$ (resp. $\exp (N) \leftrightarrow \exp \left(N^{\prime}\right)$ ), we have the isomorphism $\sigma_{\mathbb{C}} \cong \sigma_{\mathbb{C}}^{\prime}$ (resp. toric . $_{\sigma} \cong$ toric $_{\sigma^{\prime}}$ ) and the commutative diagram

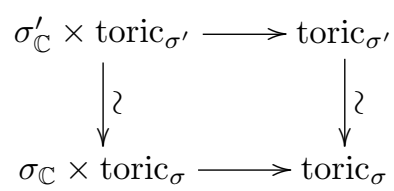

Moreover we define the $\sigma_{\mathbb{C} \text {-action }}$

$$
\sigma_{\mathbb{C}} \times E_{\sigma} \rightarrow E_{\sigma} ; \quad(w N,(s, F)) \mapsto(\exp (2 \pi \sqrt{-1} w) s, \exp (-w N) F),
$$

and the $\sigma_{\mathbb{C}^{-a c t i o n}}^{\prime}$ on $E_{\sigma^{\prime}}^{\prime}$ is defined similarly. If we consider

$$
\mathrm{Gr}_{-1}^{W}: \check{E}_{\sigma^{\prime}}^{\prime} \rightarrow \check{E}_{\sigma} ; \quad(s, F) \mapsto\left(s, \mathrm{Gr}_{-1}^{W}(F)\right),
$$

the diagram (3.1) induces the commutative diagram

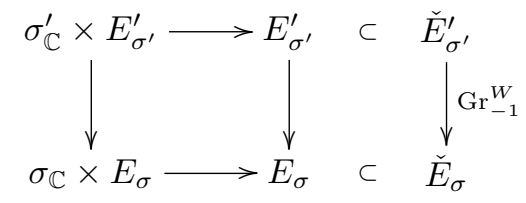

\section{$\S 3.2$. The torsor property of $E_{\sigma^{\prime}}^{\prime} \rightarrow \Gamma^{\prime}\left(\sigma^{\prime}\right)^{\mathrm{gp}} \backslash D_{\sigma^{\prime}}^{\prime}$}

Lemma 3.1. The action of $\sigma_{\mathbb{C}}^{\prime}$ on $E_{\sigma^{\prime}}^{\prime}$ is proper and free.

Proof. Since the lower horizontal action in (3.2) is free [KU, (7.2.9)], the upper horizontal action in (3.2) is also free.

The $\sigma_{\mathbb{C}^{-a c t i o n}}^{\prime}$ is proper if and only if the following condition is satisfied:

- For $x^{\prime}, y^{\prime} \in E_{\sigma^{\prime}}^{\prime}$, and sequences $\left\{x_{\lambda}^{\prime}\right\}$ in $E_{\sigma^{\prime}}^{\prime}$ and $\left\{h_{\lambda}^{\prime}\right\}$ in $\sigma_{\mathbb{C}}^{\prime}$ such that $x_{\lambda}^{\prime} \rightarrow x^{\prime}$ and $h_{\lambda}^{\prime} x_{\lambda}^{\prime} \rightarrow y^{\prime}$, there exists $h^{\prime} \in \sigma_{\mathbb{C}}^{\prime}$ such that $h_{\lambda}^{\prime} \rightarrow h^{\prime}$.

We will now show that the above condition holds. Taking $x^{\prime}, y^{\prime},\left\{x_{\lambda}^{\prime}\right\},\left\{h_{\lambda}^{\prime}\right\}$ as above, we let

$$
x:=\mathrm{Gr}_{-1}^{W}\left(x^{\prime}\right), \quad y=\operatorname{Gr}_{-1}^{W}\left(y^{\prime}\right), \quad h_{\lambda}:=\left.h_{\lambda}^{\prime}\right|_{\text {End } H_{\mathbb{Q}}} .
$$


Since the $\sigma_{\mathbb{C}}$-action is proper [KU, (7.2.2)], there exists $h \in \sigma_{\mathbb{C}}$ such that $h_{\lambda} \rightarrow h$. By the isomorphism $\sigma \cong \sigma^{\prime}$, there exists $h^{\prime} \in \sigma_{\mathbb{C}}^{\prime}$ such that $h=\left.h^{\prime}\right|_{\text {End } H_{\mathbb{Q}}}$ and $h_{\lambda}^{\prime} \rightarrow h^{\prime}$.

Lemma 3.2 ([KU, Lemma 7.3.3]). Let $H$ be a topological group and $X$ be a topological space, and assume that we have an action $H \times X \rightarrow X$ which is proper and free. Furthermore assume that the following condition is satisfied:

- For $x \in X$, there exists a topological space $S$, a morphism $\iota: S \rightarrow X$ and an open neighborhood $U$ of 1 in $H$ such that $U \times S \rightarrow X ;(h, s) \mapsto h \iota(s)$, induces an isomorphism onto an open subset of $X$.

Then $X \rightarrow H \backslash X$ is an $H$-torsor.

Proposition 3.3 ([KNU2, Theorem A(2)]). The action of $\sigma_{\mathbb{C}}^{\prime}$ on $E_{\sigma^{\prime}}^{\prime}$ satisfies the condition of Lemma 3.2. Then $E_{\sigma^{\prime}}^{\prime} \rightarrow \Gamma^{\prime}\left(\sigma^{\prime}\right)^{\mathrm{gp}} \backslash D_{\sigma^{\prime}}^{\prime}$ is a $\sigma_{\mathbb{C}}^{\prime}$-torsor.

Proof. Since $\sigma^{\prime}(s)_{\mathbb{C}} \hookrightarrow T_{\check{D}^{\prime}}(F)$ for $(s, F) \in E_{\sigma^{\prime}}^{\prime}$ (in this case $\sigma^{\prime}(s)=\sigma^{\prime}$ if $s=0$, and $\sigma^{\prime}(s)=0$ otherwise), the proof is the same as for the pure case [KU, (7.3.5)].

Since $p_{1}: E_{\sigma} \rightarrow \Gamma(\sigma)^{\mathrm{gP}} \backslash D_{\sigma}$ (resp. $p_{1}^{\prime}: E_{\sigma^{\prime}}^{\prime} \rightarrow \Gamma^{\prime}\left(\sigma^{\prime}\right)^{\mathrm{gp}} \backslash D_{\sigma^{\prime}}^{\prime}$ ) is a $\sigma_{\mathbb{C}^{-}}$torsor $\left(\sigma_{\mathbb{C}}^{\prime}\right.$-torsor), the diagram (3.2) induces the following property:

Corollary 3.4. The commutative diagram

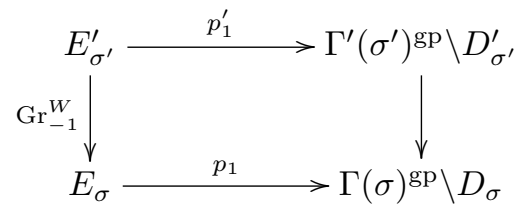

is Cartesian.

\section{§3.3. Limiting Hodge filtrations and liftings of the period map}

Let $\tilde{\phi}$ be a local lifting of the period map $\phi$. Then we have the holomorphic map

$$
\hat{\phi}: \Delta^{*} \rightarrow \check{D} ; \quad s \mapsto \exp (-l(s) N) \tilde{\phi}(s) .
$$

We call this an untwisted period map. By [Sc], this map can be extended over $\Delta$. We denote $\hat{\phi}(0)$ by $F_{\tilde{\phi}}$. Remark that $F_{\tilde{\phi}}$ depends upon the choice of the local lifting $\tilde{\phi}$. The untwisted map $\hat{\phi}$ gives the lifting

$$
\Delta \rightarrow E_{\sigma} ; \quad s \mapsto(s, \hat{\phi}(s)),
$$


of $\phi$. This gives the following diagram:

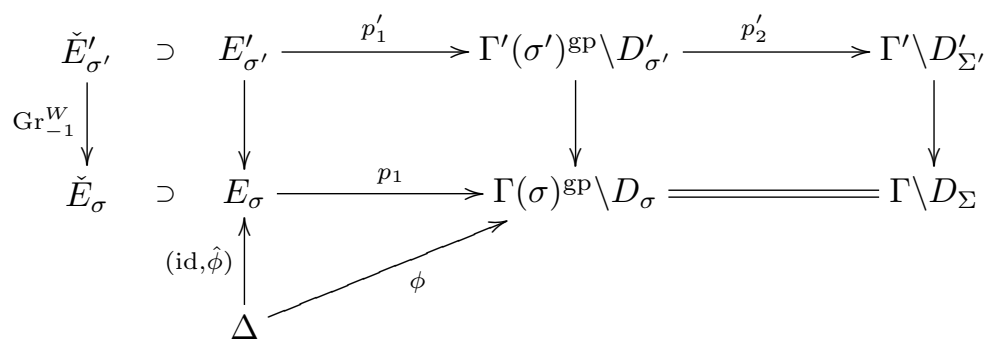

for $\sigma^{\prime} \in \Sigma^{\prime}$ such that $\sigma^{\prime} \neq\{0\}$.

For $(s, F) \in \check{E}_{\sigma^{\prime}}^{\prime}$ such that $\operatorname{Gr}_{-1}^{W}(F)=F_{\hat{\phi}(s)}$, we have the exact sequence

$$
0 \rightarrow F_{\hat{\phi}(s)}^{p} \rightarrow F^{p} \rightarrow \mathbb{C} \rightarrow 0
$$

if $p \leq 0$, and $F_{\hat{\phi}(s)}^{p} \cong F^{p}$ otherwise. Then

$$
F^{p}= \begin{cases}\mathbb{C}(z, 1)+F_{\hat{\phi}(s)}^{p} & \text { if } p \leq 0, \\ F_{\hat{\phi}(s)}^{p} & \text { if } p>0,\end{cases}
$$

where $(z, 1) \in H_{\mathbb{C}}^{\prime}$ is represented with respect to the decomposition (2.2). By the admissibility condition (2.10), a generator of $\sigma^{\prime} \in \Sigma^{\prime}$ can be written as

$$
N^{\prime}=\left(\begin{array}{cc}
N & N a \\
0 & 0
\end{array}\right)
$$

for some $a \in H_{\mathbb{Q}}$.

Proposition 3.5. For $(s, F) \in \check{E}_{\sigma^{\prime}}^{\prime}$ such that $\operatorname{Gr}_{-1}^{W}(F)=F_{\hat{\phi}(s)}$,

$$
(s, F) \in E_{\sigma^{\prime}}^{\prime} \Leftrightarrow \begin{cases}z \in H_{\mathbb{C}} & \text { if } s \neq 0, \\ z+a \in F_{\tilde{\phi}}^{0}+\operatorname{Ker}(N) & \text { if } s=0,\end{cases}
$$

where $z \in H_{\mathbb{C}}$ is as in (3.6).

Proof. If $s \neq 0$, then

$$
\operatorname{Gr}_{-1}^{W}\left(\exp \left(l(s) N^{\prime}\right) F\right)=\exp (l(s) N) \hat{\phi}(s)=\tilde{\phi}(s) \in D
$$

for any $z \in H_{\mathbb{C}}$. Then $(s, F) \in E_{\sigma^{\prime}}^{\prime}$ for any $z \in H_{\mathbb{C}}$.

If $s=0$, then

$$
N(z+a) \in F_{\tilde{\phi}}^{-1}
$$

by the transversality condition for nilpotent orbits. Since $\left(F_{\tilde{\phi}}, W(N)\right)$ is a MHS and $N$ is a $(-1,-1)$-morphism, $N(z+a) \in F_{\tilde{\phi}}^{-1}$ if $z+a \in F_{\tilde{\phi}}^{0}+\operatorname{Ker}(N)$. 


\section{§. A bijection}

In this section, we define a bijective map between $J^{\mathrm{KNU}}$ and $J^{\mathrm{GGK}}$ as sets.

\section{§4.1. A map from $J^{\mathrm{GGK}}$ to $J^{\mathrm{KNU}}$}

Let $\nu$ be an ANF. It defines an AVMHS

$$
\nu: \Delta^{*} \rightarrow \Gamma^{\prime} \backslash D^{\prime} .
$$

Taking a local lifting $\tilde{\nu}$ of $\nu$, we have the local lifting $\tilde{\phi}=\operatorname{Gr}_{-1}^{W}(\tilde{\nu})$ of $\phi$. Let $N^{\prime}$ be the logarithm of monodromy of $\tilde{\nu}$. Similarly to (3.4), we define

$$
\hat{\nu}: \Delta^{*} \rightarrow \check{D}^{\prime} ; \quad s \mapsto \exp \left(-l(s) N^{\prime}\right) \tilde{\nu}(s) .
$$

By the admissibility condition (2.6), $\hat{\nu}$ extends over $\Delta$ and $\sigma^{\prime}=\mathbb{R}_{\geq 0} N$ is in $\Sigma^{\prime}$. We denote $\hat{\nu}(0)$ by $F_{\tilde{\nu}}$. By $[\mathrm{P}],\left(\sigma^{\prime}, F_{\tilde{\nu}}\right)$ is a nilpotent orbit. We have the commutative diagram

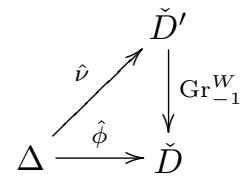

that is, $\hat{\nu}$ is a lifting of $\hat{\phi}$.

We fix $F_{\tilde{\nu}}$ as a reference point of $\check{D}^{\prime}$. By Proposition 2.2, the vertical morphism of the above diagram is a fiber bundle with fiber $\mathfrak{h} /(\mathfrak{h} \cap \mathfrak{b})$. Recall that

$$
\begin{aligned}
& \mathfrak{h}=\left\{X \in \operatorname{End}\left(H_{\mathbb{C}}^{\prime}\right)|X|_{\operatorname{End}\left(H_{\mathbb{C}}\right)}=0, X(e) \in H_{\mathbb{C}}\right\}, \\
& \mathfrak{h} \cap \mathfrak{b}=\left\{X \in \mathfrak{h} \mid X(e) \in F_{\tilde{\phi}}^{0}\right\}, \\
& \left.V=\bigoplus_{p<0} I^{p, q} \quad \text { (i.e., } F_{\tilde{\phi}}^{0} \oplus V=H_{\mathbb{C}}\right),
\end{aligned}
$$

and then

$$
\mathfrak{h} /(\mathfrak{h} \cap \mathfrak{b}) \cong V ; \quad X_{v} \leftrightarrow v,
$$

where $X_{v} \in \mathfrak{h}$ is such that $X_{v}(e)=v$. Taking a boundary point $\left((0, \dot{v}),[\nu]_{0}\right) \in$ $J^{\mathrm{GGK}}$ where

$$
\dot{v}=v \bmod H_{\mathbb{Z}} \cap \operatorname{Ker}(N)
$$

for some $v \in V \cap \operatorname{Ker}(N)$, we define

$$
\alpha\left((0, \dot{v}),[\nu]_{0}\right):=\left(0,\left(\sigma^{\prime}, \exp \left(\sigma_{\mathbb{C}}^{\prime}\right) \exp \left(X_{-v}\right) F_{\tilde{\nu}}\right)\right) .
$$

By Proposition $3.5, \alpha\left((0, \dot{v}),[\nu]_{0}\right)$ is in $J^{\mathrm{KNU}}$. 
Lemma 4.1. $\alpha\left((0, \dot{v}),[\nu]_{0}\right)$ is well-defined.

Proof. We show that $\alpha\left((0, \dot{v}),[\nu]_{0}\right)$ does not depend on the choice of $v$ of (4.3), a lifting $\tilde{\nu}$ and a representative $\left((0, \dot{v}),[\nu]_{0}\right)$.

First, we take $x \in H_{\mathbb{Z}} \cap \operatorname{Ker}(N)$. By (2.11), this gives $\operatorname{Ad}\left(\gamma_{x}\right) N^{\prime}=N^{\prime}$ for

$$
\gamma_{x}=\left(\begin{array}{ll}
I & x \\
0 & 1
\end{array}\right) \in \Gamma^{\prime}
$$

Then

$$
\begin{aligned}
\left(\sigma^{\prime}, \exp \left(\sigma_{\mathbb{C}}^{\prime}\right) \exp \left(X_{-v+x}\right) F_{\tilde{\nu}}\right) & =\left(\sigma^{\prime}, \exp \left(\sigma_{\mathbb{C}}^{\prime}\right) \gamma_{x} \exp \left(X_{-v}\right) F_{\tilde{\nu}}\right) \\
& =\gamma_{x}\left(\sigma^{\prime}, \exp \left(\sigma_{\mathbb{C}}^{\prime}\right) \exp \left(X_{-v}\right) F_{\tilde{\nu}}\right)
\end{aligned}
$$

Next, we take another lifting $\gamma \tilde{\nu}$ for $\gamma \in \Gamma^{\prime}$. The monodromy cone that arises from $\gamma \tilde{\nu}$ is $\operatorname{Ad}(\gamma) \sigma^{\prime}$ and $F_{\gamma \tilde{\nu}}=\gamma F_{\tilde{\nu}}$. Since $v \in \operatorname{Ker}(N)$, we have

$$
\exp \left(X_{-v}\right) \gamma=\gamma \exp \left(X_{-v}\right)
$$

Then

$\left(\operatorname{Ad}(\gamma) \sigma_{\mathbb{C}}^{\prime}, \exp \left(\operatorname{Ad}(\gamma) \sigma_{\mathbb{C}}^{\prime}\right) \exp \left(X_{-v}\right) F_{\gamma \tilde{\nu}}\right)=\gamma\left(\sigma^{\prime}, \exp \left(\sigma_{\mathbb{C}}^{\prime}\right) \exp \left(X_{-v}\right) F_{\tilde{\nu}}\right)$

Finally, we take $\left(\left(0, \dot{v}_{1}\right),\left[\nu_{1}\right]_{0}\right) \sim\left(\left(0, \dot{v}_{2}\right),\left[\nu_{2}\right]_{0}\right)$ and let

$$
F_{\tilde{\nu}_{i}}^{p}=\left\{\begin{array}{ll}
\mathbb{C}\left(z_{i}, 1\right)+F_{\tilde{\phi}}^{p} & \text { if } p \leq 0, \\
F_{\tilde{\phi}}^{p} & \text { if } p>0,
\end{array} \quad \text { for } i=1,2,\right.
$$

where $\tilde{\nu}_{i}$ are local liftings. Let $\mu=\nu_{1}-\nu_{2}$. Then there exists a local lifting $\tilde{\mu}$ such that

$$
F_{\tilde{\mu}}^{p}= \begin{cases}\mathbb{C}\left(z_{1}-z_{2}, 1\right)+F_{\tilde{\phi}}^{p} & \text { if } p \leq 0 \\ F_{\tilde{\phi}}^{p} & \text { if } p>0\end{cases}
$$

Since $\left(\left(0, \dot{v}_{1}\right),\left[\nu_{1}\right]_{0}\right) \sim\left(\left(0, \dot{v}_{2}\right),\left[\nu_{2}\right]_{0}\right), \mu$ is a NF such that $\mu(0)=\dot{v}_{1}-\dot{v}_{2} \in J_{0}^{\mathrm{GGK}, 0}$. Then there exist $v_{1}, v_{2} \in \operatorname{Ker}(N) \cap V$ such that

$$
\dot{v}_{i}=v_{i} \bmod H_{\mathbb{Z}} \cap \operatorname{Ker}(N)
$$

and $z_{1}-z_{2}=v_{1}-v_{2}$.

On the other hand, the logarithm of the monodromy of $\tilde{\nu}_{i}$ is described by

$$
\left(\begin{array}{cc}
N & N a_{i} \\
0 & 0
\end{array}\right)
$$


for some $a_{i} \in H_{\mathbb{Q}}$. Then the logarithm of the monodromy of $\tilde{\mu}$ is

$$
\left(\begin{array}{cc}
N & N\left(a_{1}-a_{2}\right) \\
0 & 0
\end{array}\right)
$$

Since $\mu$ is a NF, $(T-I)\left(a_{1}-a_{2}\right) \in(T-I) H_{\mathbb{Z}}$ by the exact sequence (2.7). Then $a_{1}-a_{2} \in H_{\mathbb{Z}}$. Setting

$$
\gamma_{a_{1}-a_{2}}=\left(\begin{array}{cc}
I & a_{1}-a_{2} \\
0 & 1
\end{array}\right)
$$

we have

$$
\operatorname{Ad}\left(\gamma_{a_{1}-a_{2}}\right)\left(\begin{array}{cc}
N & N a_{2} \\
0 & 0
\end{array}\right)=\left(\begin{array}{cc}
N & N a_{1} \\
0 & 0
\end{array}\right)
$$

by (2.11). Since $\alpha\left(\left(0, \dot{v}_{2}\right),\left[\nu_{2}\right]_{0}\right)$ does not depend on the choice of lifting, we may take $\gamma_{a_{1}-a_{2}} \tilde{\nu}_{2}$ as a lifting of $\nu_{2}$. The monodromy cone that arises from $\gamma_{a_{1}-a_{2}} \tilde{\nu}_{2}$ is $\sigma^{\prime}$. Then

$$
\begin{aligned}
\left(\sigma^{\prime}, \exp \left(\sigma_{\mathbb{C}}^{\prime}\right) \exp \left(X_{-v_{2}}\right) F_{\tilde{\nu}_{2}}\right) & =\left(\sigma^{\prime}, \exp \left(\sigma_{\mathbb{C}}^{\prime}\right) \exp \left(X_{-v_{2}}\right) \exp \left(X_{v_{2}-v_{1}}\right) F_{\tilde{\nu}_{1}}\right) \\
& =\left(\sigma^{\prime}, \exp \left(\sigma_{\mathbb{C}}^{\prime}\right) \exp \left(X_{-v_{1}}\right) F_{\tilde{\nu}_{1}}\right)
\end{aligned}
$$

Therefore, $\alpha$ defines a map $\alpha: J^{\mathrm{GGK}} \rightarrow J^{\mathrm{KNU}}$ where the restriction $\left.\alpha\right|_{J}$ is canonical.

\section{§4.2. A map from $J^{\mathrm{KNU}}$ to $J^{\mathrm{GGK}}$}

Let $\tilde{\phi}$ be a lifting of $\phi$. By Corollary 3.4, for $\left(0,\left(\sigma^{\prime}, Z\right)\right) \in J_{\sigma^{\prime}}$, we have $(0, F) \in E_{\sigma^{\prime}}^{\prime}$ such that

$$
\mathrm{Gr}_{-1}^{W}(0, F)=\left(0, F_{\tilde{\phi}}\right), \quad p_{1}^{\prime}(0, F)=\left(\sigma^{\prime}, Z\right) .
$$

We denote this filtration by $F_{\left(\sigma^{\prime}, Z\right), \tilde{\phi}}$.

Lemma 4.2. For $\gamma \in \Gamma^{\prime}$ such that $\left.\gamma\right|_{\text {Aut } H_{\mathbb{Z}}}=T^{n}, \gamma \exp \left((m-n) N^{\prime}\right) F_{\left(\sigma^{\prime}, Z\right), \tilde{\phi}}=$ $F_{\gamma\left(\sigma^{\prime}, Z\right), T^{m} \tilde{\phi}}$.

Proof. By Proposition 3.5, there exists $x \in \operatorname{Ker}(N)$ such that

$$
F_{\left(\sigma^{\prime}, Z\right), \tilde{\phi}}^{p}= \begin{cases}\mathbb{C}(x-a, 1)+F_{\tilde{\phi}}^{p} & \text { if } p \leq 0 \\ F_{\tilde{\phi}}^{p} & \text { if } p>0 .\end{cases}
$$

Writing $\gamma=\left(\begin{array}{cc}T^{n} & b \\ 0 & 1\end{array}\right)$ for some $b \in H_{\mathbb{Z}}$, we have

$$
\gamma \exp \left((m-n) N^{\prime}\right) F_{\left(\sigma^{\prime}, Z\right), \tilde{\phi}}^{p}= \begin{cases}\mathbb{C}\left(T^{m} x-T^{n} a+b, 1\right)+F_{T^{m} \tilde{\phi}}^{p} & \text { if } p \leq 0 \\ F_{T^{m} \tilde{\phi}}^{p} & \text { if } p>0\end{cases}
$$


Since $x \in \operatorname{Ker}(N)$,

$$
T^{m} x-T^{n} a+b=x-\left(T^{n} a-b\right) .
$$

By (2.11) and Proposition 3.5, $\left(0, \gamma \exp \left((m-n) N^{\prime}\right) F_{\left(\sigma^{\prime}, Z\right), \tilde{\phi}}\right) \in E_{\operatorname{Ad}(\gamma) \sigma^{\prime}}^{\prime}$, which satisfies

$$
\begin{aligned}
& \operatorname{Gr}_{-1}^{W}\left(0, \gamma \exp \left((m-n) N^{\prime}\right) F_{\left(\sigma^{\prime}, Z\right), \tilde{\phi}}\right)=\left(0, F_{T^{m} \tilde{\phi}}\right), \\
& p_{1}^{\prime}\left(0, \gamma \exp \left((m-n) N^{\prime}\right) F_{\left(\sigma^{\prime}, Z\right), \tilde{\phi}}\right)=\gamma\left(\sigma^{\prime}, Z\right) .
\end{aligned}
$$

Let $\hat{\phi}: \Delta \rightarrow \check{D}$ be the untwisted period map. Since $\check{D}^{\prime} \rightarrow \check{D}$ is a fiber bundle, there exists a lifting of $\hat{\phi}$,

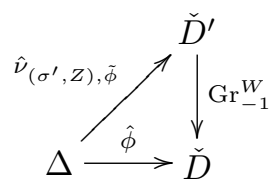

such that $\hat{\nu}_{\left(\sigma^{\prime}, Z\right), \tilde{\phi}}(0)=F_{\left(\sigma^{\prime}, Z\right), \tilde{\phi}}$, after shrinking $\Delta$ if necessary. We then have a holomorphic map

$$
\Delta^{*} \rightarrow \Gamma^{\prime} \backslash D^{\prime} ; \quad s \mapsto p_{2}^{\prime} \circ p_{1}^{\prime}\left(s, \hat{\nu}_{\left(\sigma^{\prime}, Z\right), \tilde{\phi}}(s)\right),
$$

which defines an AVMHS, i.e., an ANF. Denoting this ANF by $\nu_{\left(\sigma^{\prime}, Z\right), \tilde{\phi}}$, we define

$$
\beta\left(0,\left(\sigma^{\prime}, Z\right)\right):=\left((0,0),\left[\nu_{\left(\sigma^{\prime}, Z\right), \tilde{\phi}}\right]_{0}\right) \in J^{\mathrm{GGK}} .
$$

Lemma 4.3. $\beta\left(0,\left(\sigma^{\prime}, Z\right)\right)$ is well-defined.

Proof. We show that $\beta\left(0,\left(\sigma^{\prime}, Z\right)\right)$ does not depend on the choice of $\hat{\nu}_{\left(\sigma^{\prime}, Z\right), \tilde{\phi}},\left(\sigma^{\prime}, Z\right)$ and $\tilde{\phi}$.

If we take liftings $\hat{\nu}_{\left(\sigma^{\prime}, Z\right), \tilde{\phi}}$ and $\hat{\nu}_{\left(\sigma^{\prime}, Z\right), \tilde{\phi}}^{\prime}$ such that

$$
\hat{\nu}_{\left(\sigma^{\prime}, Z\right), \tilde{\phi}}(0)=\hat{\nu}_{\left(\sigma^{\prime}, Z\right), \tilde{\phi}}^{\prime}(0)=F_{\left(\sigma^{\prime}, Z\right), \tilde{\phi}},
$$

then $\mu:=\nu_{\left(\sigma^{\prime}, Z\right), \tilde{\phi}}-\nu_{\left(\sigma^{\prime}, Z\right), \tilde{\phi}}^{\prime}$ is a NF and $\mu(0)=0 \in J_{0}^{\mathrm{GGK}, 0}$. Then

$$
\left((0,0),\left[\nu_{\left(\sigma^{\prime}, Z\right), \tilde{\phi}}\right]_{0}\right) \sim\left((0,0),\left[\nu_{\left(\sigma^{\prime}, Z\right), \tilde{\phi}}^{\prime}\right]_{0}\right) .
$$

Moreover, by Lemma 4.2 ,

$$
\gamma \exp \left((m-n) N^{\prime}\right) \hat{\nu}_{\left(\sigma^{\prime}, Z\right), \tilde{\phi}}(0)=F_{\gamma\left(\sigma^{\prime}, Z\right), T^{m}} \tilde{\phi} .
$$

If we take $\hat{\nu}_{\gamma\left(\sigma^{\prime}, Z\right), T^{m} \tilde{\phi}}=\gamma \exp \left((m-n) N^{\prime}\right) \hat{\nu}_{\left(\sigma^{\prime}, Z\right), \tilde{\phi}}$ as a lifting of $T^{m} \hat{\phi}$, then $\nu_{\left(\sigma^{\prime}, Z\right), \tilde{\phi}}=\nu_{\gamma\left(\sigma^{\prime}, Z\right), T^{m}} \tilde{\phi}^{\cdot}$ 
Thus $\beta$ defines a map $\beta: J^{\mathrm{KNU}} \rightarrow J^{\mathrm{GGK}}$ where the restriction $\left.\beta\right|_{J}$ is canonical.

Proposition 4.4. $\alpha=\beta^{-1}$ and $\beta=\alpha^{-1}$, i.e., $J^{\mathrm{GGK}}$ is bijective to $J^{\mathrm{KNU}}$.

Proof. For $\left((0, \dot{v}),[\nu]_{0}\right) \in J^{\mathrm{GGK}}$, we set $\left(0,\left(\sigma^{\prime}, Z\right)\right):=\alpha\left((0, \dot{v}),[\nu]_{0}\right)$. By making a suitable choice of $\tilde{\nu}, \tilde{\phi}$ and $v$, we have $F_{\left(\sigma^{\prime}, Z\right), \tilde{\phi}}=\exp \left(X_{-v}\right) F_{\tilde{\nu}}$. Therefore $\mu(0)=\dot{v}$ for $\mu=\nu-\nu_{\left(\sigma^{\prime}, Z\right), \tilde{\phi}}$, which implies

$$
\left((0, \dot{v}),[\nu]_{0}\right) \sim\left((0,0),\left[\nu_{\left(\sigma^{\prime}, Z\right), \tilde{\phi}}\right]_{0}\right)=\beta\left(0,\left(\sigma^{\prime}, Z\right)\right) .
$$

On the other hand, for $\left(0,\left(\sigma^{\prime}, Z\right)\right) \in J^{\mathrm{KNU}}$, we set $\left((0,0),[\nu]_{0}\right):=\beta\left(0,\left(\sigma^{\prime}, Z\right)\right)$. By a suitable choice of $\tilde{\nu},\left(\sigma^{\prime}, Z\right)$ and $\tilde{\phi}$, we have $F_{\tilde{\nu}}=F_{\left(\sigma^{\prime}, Z\right), \tilde{\phi}}$. Therefore,

$$
\left(0,\left(\sigma^{\prime}, Z\right)\right)=\left(0,\left(\sigma^{\prime}, \exp \left(\sigma_{\mathbb{C}}^{\prime}\right) F_{\tilde{\nu}}\right)\right)=\alpha\left((0,0),[\nu]_{0}\right) .
$$

\section{§5. A homeomorphism}

In this section, we show the following main theorem:

Theorem 5.1. $J^{\mathrm{GGK}}$ is homeomorphic to $J^{\mathrm{KNU}}$.

To show continuity, we describe an open neighborhood in $J^{\mathrm{KNU}}$. We recall that the topology on $J^{\mathrm{KNU}}$ is induced from $K_{\sigma}$ through the following diagram:

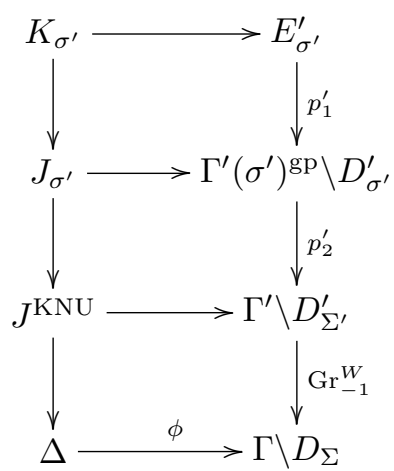

We describe an open neighborhood in $J^{\mathrm{KNU}}$ using the following steps:

Step 1. Describe an open neighborhood in $E_{\sigma^{\prime}}^{\prime}$.

Step 2. Describe an open neighborhood in $\Gamma^{\prime}\left(\sigma^{\prime}\right)^{\mathrm{gp}} \backslash D_{\sigma^{\prime}}^{\prime}$.

Step 3. Describe an open neighborhood in $J^{\mathrm{KNU}}$.

Open neighborhoods in $J^{\mathrm{GGK}}$ are described in (2.8). Comparing these, we show that the bijection constructed in the last section is continuous. 


\section{$\S 5.1$. Proof of the main theorem}

Setting. We take a boundary point $\left(0,\left(\sigma^{\prime}, Z\right)\right) \in J^{\mathrm{KNU}}$. Choosing a lifting $\tilde{\phi}$ of the period map $\phi$, we have the untwisted period map $\hat{\phi}: \Delta \rightarrow \check{D}$. Since $\sigma_{\mathbb{C}} \hookrightarrow$ $T_{\check{D}}\left(F_{\tilde{\phi}}\right)$, we may take a $\mathbb{C}$-subspace $B$ of $\mathfrak{g}_{\mathbb{C}}$ such that $B \oplus \sigma_{\mathbb{C}} \cong T_{\check{D}}\left(F_{\tilde{\phi}}\right)$. An open neighborhood at $F_{\tilde{\phi}}$ in $\check{D}$ is described by

$$
\left\{\exp \left(a_{1}\right) \exp \left(a_{2}\right) F_{\tilde{\phi}} \mid a_{1} \in U_{1}, a_{2} \in U_{2}\right\} \cong U_{1} \times U_{2}
$$

where $U_{1}$ (resp. $U_{2}$ ) is a sufficiently small open neighborhood of 0 in $\sigma_{\mathbb{C}}$ (resp. $B$ ). We assume that the image of $\hat{\phi}$ is included in this open neighborhood, after shrinking $\Delta$ if necessary. We put $\hat{\phi}(s)=\left(\hat{\phi}_{1}(s), \hat{\phi}_{2}(s)\right)$, where $\hat{\phi}_{1}: \Delta \rightarrow \sigma_{\mathbb{C}} \cong \mathbb{C}$ is a holomorphic function such that $\hat{\phi}_{1}(0)=0$. By using the coordinate $t=$ $\exp \left(2 \pi \sqrt{-1} \hat{\phi}_{1}(s)\right) \cdot s$ on $\Delta$ the untwisted period map is

$$
\hat{\phi}(t)=\exp (-l(t) N) \tilde{\phi}(t)=\exp \left(-\hat{\phi}_{1}(s) N-l(s) N\right) \tilde{\phi}(t)=\exp \left(-\hat{\phi}_{1}(s) N\right) \hat{\phi}(s) .
$$

Then $\hat{\phi}_{1}(t)=0$ for $\hat{\phi}(t)=\left(\hat{\phi}_{1}(t), \hat{\phi}_{2}(t)\right) \in U_{1} \times U_{2}$. It is significant that $F_{\tilde{\phi}}, F_{\tilde{\nu}}$ and $F_{\left(\sigma^{\prime}, Z\right), \tilde{\phi}}$ do not depend on this coordinate change (i.e., the bijection $\alpha$ is independent).

Steps 1 and 2. In the pure case, neighborhoods in $E_{\sigma}$ and in $\Gamma(\sigma)^{\mathrm{gp}} \backslash D_{\sigma}$ are described in [KU, (7.3.5)]. We describe neighborhoods in $E_{\sigma^{\prime}}^{\prime}$ and in $\Gamma^{\prime}\left(\sigma^{\prime}\right)^{\mathrm{gp}} \backslash D_{\sigma^{\prime}}^{\prime}$ in a similar way. Now we have the point $\left(0, F_{\left(\sigma^{\prime}, Z\right), \tilde{\phi}}\right) \in E_{\sigma^{\prime}}^{\prime}$ as described in (4.4). Since $\mathrm{Gr}_{-1}^{W}: \check{D}^{\prime} \rightarrow \check{D}$ is a fiber bundle with fiber $V$, we have a local trivialization

$$
\left(\mathrm{Gr}_{-1}^{W}\right)^{-1}\left(U_{1} \times U_{2}\right) \cong U_{1} \times U_{2} \times V .
$$

Since $F_{\left(\sigma^{\prime}, Z\right), \tilde{\phi}} \in\left(\mathrm{Gr}_{-1}^{W}\right)^{-1}\left(F_{\tilde{\phi}}\right)$, we can assume that $(0,0,0)$ corresponds to $F_{\left(\sigma^{\prime}, Z\right), \tilde{\phi}}$. Using this local trivialization, an open neighborhood at $\left(0, F_{\left(\sigma^{\prime}, Z\right), \tilde{\phi}}\right)$ in $\check{E}_{\sigma^{\prime}}^{\prime}$ can be described by

$$
\left\{\left(a_{0},\left(a_{1}, a_{2}, v\right)\right) \mid a_{0} \in U_{0}, a_{1} \in U_{1}, a_{2} \in U_{2}, v \in U_{3}\right\}
$$

where $U_{0}$ (resp. $U_{3}$ ) is a sufficiently small open neighborhood of 0 in toric $\sigma_{\sigma^{\prime}}$ $($ resp. $V)$. Let

$$
A^{\prime}=\left\{\left(a_{0},\left(0, a_{2}, v\right)\right) \mid a_{0} \in U_{0}, a_{2} \in U_{2}, v \in U_{3}\right\}, \quad S^{\prime}=A^{\prime} \cap E_{\sigma^{\prime}}^{\prime} .
$$

Using the diagram (3.2), the $\sigma_{\mathbb{C}}^{\prime}$-action defines an open inclusion map

$$
U_{1} \times S^{\prime} \hookrightarrow E_{\sigma^{\prime}}^{\prime} .
$$

This inclusion map induces the open inclusion map

$$
\sigma_{\mathbb{C}}^{\prime} \times S^{\prime} \hookrightarrow E_{\sigma^{\prime}}^{\prime},
$$


after shrinking $S^{\prime}$ if necessary. Then $p_{1}^{\prime}\left(S^{\prime}\right)$ is an open set of $\Gamma^{\prime}\left(\sigma^{\prime}\right)^{\mathrm{gp}} \backslash D_{\sigma^{\prime}}^{\prime}$ and $p_{1}^{\prime}\left(S^{\prime}\right) \cong S^{\prime}$. Moreover, $p_{2}^{\prime} \circ p_{1}^{\prime}\left(S^{\prime}\right)$ is an open neighborhood of $\left(\sigma^{\prime}, Z\right)$ in $\Gamma^{\prime} \backslash D_{\Sigma^{\prime}}^{\prime}$.

Step 3. Since $p_{1}^{\prime}\left(S^{\prime}\right)$ (resp. $\left.p_{2}^{\prime} \circ p_{1}^{\prime}\left(S^{\prime}\right)\right)$ is an open neighborhood in $\Gamma^{\prime}\left(\sigma^{\prime}\right)^{\mathrm{gp}} \backslash D_{\sigma^{\prime}}^{\prime}$ (resp. $\left.\Gamma^{\prime} \backslash D_{\Sigma^{\prime}}^{\prime}\right), p_{1}^{\prime}\left(\left(\Delta \times S^{\prime}\right) \cap K_{\sigma^{\prime}}\right)$ (resp. $\left.p_{2}^{\prime} \circ p_{1}^{\prime}\left(\left(\Delta \times S^{\prime}\right) \cap K_{\sigma^{\prime}}\right)\right)$ is an open neighborhood of $J_{\sigma^{\prime}}\left(\right.$ resp. $\left.J^{\mathrm{KNU}}\right)$. Moreover, since $p_{1}^{\prime}\left(S^{\prime}\right) \cong S^{\prime}$,

$$
p_{1}^{\prime}\left(\left(\Delta \times S^{\prime}\right) \cap K_{\sigma^{\prime}}\right) \cong\left(\Delta \times S^{\prime}\right) \cap K_{\sigma^{\prime}} .
$$

We describe $\left(\Delta \times S^{\prime}\right) \cap K_{\sigma^{\prime}}$ explicitly. By (3.5), we have the commutative diagram

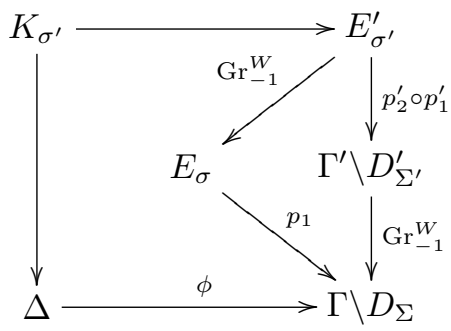

Then, for $(t, \xi) \in \Delta \times E_{\sigma^{\prime}}^{\prime},(t, \xi) \in K_{\sigma^{\prime}}$ if, and only if,

$$
\phi(t)=\operatorname{Gr}_{-1}^{W} \circ p_{2}^{\prime} \circ p_{1}^{\prime}(\xi)=p_{1} \circ \operatorname{Gr}_{-1}^{W}(\xi) .
$$

Lemma 5.2. $\left(\left(p_{1}\right)^{-1}(\phi(t))\right) \cap \operatorname{Gr}_{-1}^{W}\left(S^{\prime}\right)=(t, \hat{\phi}(t))$.

Proof. Since $p_{1}((t, \hat{\phi}(t)))=\phi(t)$ and $p_{1}$ is a $\sigma_{\mathbb{C}}$-torsor, the fiber is

$$
\left(p_{1}\right)^{-1}(\phi(t))=\sigma_{\mathbb{C}} \cdot(t, \hat{\phi}(t))=\{(\exp (2 \pi \sqrt{-1} x) t, \exp (-x N) \hat{\phi}(t)) \mid x \in \mathbb{C}\} .
$$

The intersection with $U_{0} \times U_{1} \times U_{2}$ is

$$
\begin{aligned}
\left(U_{0}\right. & \left.\times U_{1} \times U_{2}\right) \cap\left(p_{1}\right)^{-1}(\phi(t)) \\
& =\left\{\left(\exp \left(2 \pi \sqrt{-1} a_{1}\right) t,-a_{1}, \hat{\phi}_{2}(t)\right) \mid \exp \left(2 \pi \sqrt{-1} a_{1}\right) t \in U_{0},-a_{1} \in U_{1}\right\} .
\end{aligned}
$$

On the other hand, for $\left(a_{0}, 0, a_{2}, v\right) \in S^{\prime}$,

$$
\operatorname{Gr}_{-1}^{W}\left(\left(a_{0}, 0, a_{2}, v\right)\right)=\left(a_{0}, 0, a_{2}\right) .
$$

Thus $\left(a_{0}, 0, a_{2}\right) \in\left(p_{1}\right)^{-1}(\phi(t))$ if, and only if, $a_{0}=t$ and $a_{2}=\hat{\phi}_{2}(t)$.

\section{Lemma 5.3.}

$$
\left(\Delta \times S^{\prime}\right) \cap K_{\sigma^{\prime}}=\left\{\begin{array}{l|l}
\left(t,\left(t, 0, \hat{\phi}_{2}(t), v\right)\right) & \begin{array}{l}
t \in U_{0} \cap \Delta \\
v \in \operatorname{Ker}(N) \cap U_{3} \text { if } t=0, \\
v \in U_{3} \text { if } t \neq 0
\end{array}
\end{array}\right\} .
$$


Proof. By Lemma 5.2, for $\left(a_{0}, 0, a_{2}, v\right) \in S^{\prime}$,

$$
\phi(t)=p_{1} \circ \mathrm{Gr}_{W}^{-1}\left(\left(a_{0}, 0, a_{2}, v\right)\right) \Rightarrow a_{0}=t \text { and } a_{2}=\hat{\phi}_{2}(t) .
$$

By Proposition 3.5, if $t \neq 0$, then $\left(t, 0, \hat{\phi}_{2}(t), v\right) \in S^{\prime}$ for $v \in U_{3}$. If $t=0$, since $(0,0, v) \in U_{1} \times U_{2} \times U_{3}$ corresponds to $\exp \left(X_{v}\right) F_{\left(\sigma^{\prime}, Z\right), \tilde{\phi}}$, we have $(0,0,0, v) \in S^{\prime}$ for $v \in F_{\tilde{\phi}}^{0}+\operatorname{Ker}(N)$. Since $V \oplus F_{\tilde{\phi}}^{0}=H_{\mathbb{C}}$ by $(2.4), v \in \operatorname{Ker}(N)$.

Homeomorphism. Let $S:=W \cap\left(\Delta \times U_{3}\right)$ where $W$ is as in (2.5) and $S$ is endowed with the strong topology in $\Delta \times U_{3}$. Then $S$ is homeomorphic to (5.3). For the local trivialization (5.1), we get

$$
\hat{\nu}: \Delta \rightarrow U_{1} \times U_{2} \times U_{3} \subset \check{D}^{\prime} ; \quad t \mapsto\left(0, \hat{\phi}_{2}(t), 0\right) .
$$

Then we have an ANF

$$
\nu: \Delta \rightarrow \Gamma^{\prime} \backslash D_{\Sigma^{\prime}}^{\prime} ; \quad t \mapsto p_{2}^{\prime} \circ p_{1}^{\prime}(t, \hat{\nu}(t)) .
$$

Following (2.8) we define a neighborhood

$$
\dot{S}(\nu)=\left\{\left((t,-\dot{v}),[\nu]_{t}\right) \mid(t, \dot{v}) \in \dot{S}\right\}
$$

at $\alpha^{-1}\left(0,\left(\sigma^{\prime}, Z\right)\right)=\left((0,0),[\nu]_{0}\right)$ in $J^{\text {GGK }}$ where $\dot{S}$ is the image of $S$ in the quotient space $W / \sim$. Then $\alpha(\dot{S}(\nu))$ is the image of (5.3) under $p_{2}^{\prime} \circ p_{1}^{\prime}$, which is a neighborhood of $\left(0,\left(\sigma^{\prime}, Z\right)\right)$. In fact

$$
\begin{aligned}
\alpha\left((t,-\dot{v}),[\nu]_{0}\right) & = \begin{cases}\left(0, \exp \left(l(t) N^{\prime}\right) \exp \left(X_{v}\right) \hat{\nu}(t)\right) & \text { if } t \neq 0 \\
\left(\sigma^{\prime}, \exp \left(\sigma_{\mathbb{C}}^{\prime}\right) \exp \left(X_{v}\right) \hat{\nu}(0)\right) & \text { if } t=0\end{cases} \\
& =p_{2}^{\prime} \circ p_{1}^{\prime}\left(t,\left(t, 0, \hat{\phi}_{2}(t), v\right)\right) .
\end{aligned}
$$

[KU, §3.1] gives a fundamental system of neighborhoods at $(0,0)$ in $S$. It defines a fundamental system of neighborhoods at $\left((0,0),[\nu]_{0}\right)$ in $J^{\mathrm{GGK}}$, which goes to a fundamental system of neighborhoods at $\left(0,\left(\sigma^{\prime}, Z\right)\right)$ in $J^{\mathrm{KNU}}$ under $\alpha$. Therefore, $\alpha$ is a homeomorphism.

\section{Acknowledgments}

The author is grateful to Professors Kazuya Kato, Chikara Nakayama, Gregory Pearlstein, Christian Schnell and Sampei Usui for their valuable advice and warm encouragement. This research was partly supported by Grant-in-Aid for JSPS Fellows from Japan Society for the Promotion of Science and by National Science Council of Taiwan. 


\section{References}

[C] J. Carlson, Extensions of mixed Hodge structures, in Journées de géométrie algébrique d'Angers, juillet 1979, Sijthoff \& Noordhoff, 1980, 107-127. Zbl 0471.14003 MR 0605338

[GGK1] M. Green, P. Griffiths and M. Kerr, Néron models and limits of Abel-Jacobi mappings, Compos. Math. 146 (2010), 288-366. Zbl 1195.14006 MR 2601630

[GGK2] _ Néron models and boundary components for degenerations of Hodge structures of mirror quintic type, in Curves and abelian varieties, V. Alexeev (ed.), Contemp. Math. 465, Amer. Math. Soc., 2007, 71-145. Zbl 1159.14004 MR 2457736

[G] P. Griffiths, Periods of integrals on algebraic manifolds. I. Construction and properties of the modular varieties, Amer. J. Math. 90 (1968), 568-626. Zbl 0169.52303 MR 0229641

[KNU1] K. Kato, C. Nakayama and S. Usui, Log intermediate Jacobians, Proc. Japan Acad. Ser. A Math. Sci. 86 (2010), 73-78. Zbl 1200.14024 MR 2657330

[KNU2] , Moduli of log mixed Hodge structures, Proc. Japan Acad. Ser. A Math. Sci. 86 (2010), 107-112. Zbl 1209.14008 MR 2663651

[KU] K. Kato and S. Usui, Classifying space of degenerating polarized Hodge structures, Ann. of Math. Stud. 169, Princeton Univ. Press, Princeton, NJ, 2009. Zbl 1172.14002 MR 2465224

[P] G. Pearlstein, Degenerations of mixed Hodge structure, Duke Math. J. 110 (2001), 217-251. Zbl 1092.14018 MR 1865240

[Sa1] M. Saito, Admissible normal functions, J. Algebraic Geom. 5 (1996), 235-276. Zbl 0918.14018 MR 1374710

[Sa2] Hausdorff property of the Néron models of Green, Griffiths and Kerr, arXiv:0803.2771.

[Sc] W. Schmid, Variation of Hodge structure: the singularities of the period mapping, Invent. Math. 22 (1973), 211-319. Zbl 0278.14003 MR 0382272

[SI] T. Shioda and H. Inose, On singular K3 surfaces, in Complex analysis and algebraic geometry, Iwanami Shoten, Tokyo, 1977, 119-136. Z Zb 0374.14006 MR 0441982

[SZ] J. Steenbrink and S. Zucker, Variation of mixed Hodge structure. I. Invent. Math. 80 (1985), 489-542. Zbl 0626.14007 MR 0791673

[U] S. Usui, Variation of mixed Hodge structure arising from family of logarithmic deformations. II. Classifying space, Duke Math. J. 51 (1984), 851-875. Zbl 0558.14005 MR 0771384 